\title{
Pivotal Technology for the Detection of Lens' Defects Based on Image Recognition
}

\author{
Yueqiu Jiang ${ }^{*}$, Fei Xia, Hongwei Gao and Yujun Wang
}

Shenyang Ligong University, Shenyang, China

\begin{abstract}
With flaws such as scratches, dirt and pitting on the surface of the lens as the research object in this paper, the key technology of machine vision inspection based on in-depth analysis of image recognition was studied as well as the programs of extraction and quantification based on image recognition on the surface of the lens. First, for the insufficient edge destruction of the traditional de-noising method, an improved filtering algorithm has been proposed. Then, the interference of the lens frame is removed when calculating the size of the defects by using the circular region detection algorithm. And a new improved calculation method of small connectivity target based on mathematical morphology by pixels' scanning and marking has been proposed. Finally, artificial neural networks were introduced and by using BP neural network classifier, the classification of scratches and pitting was realized. Experimental results showed certain guiding significance for detecting the surface quality of the lens based on image processing technology.
\end{abstract}

Keywords: Lens defects, Machine vision, Enhancement, Segmentation, Identification

\section{INTRODUCTION}

With the rapid development of modern science and optical technology, precision optical components became more popular, especially in the industrial sector in the field of hi-tech industries. Different kinds of defects such as scratch, explosive grain, split, smudge, bubble, breach and spot would inevitably appear in the process of precision optics and fiber optic components. By multiple reflections and refractions, these beams of light will transfer everywhere in the optics system, leaving bad effects on the results of optical measure instruments [1]. And these defects also make effects on different kinds of applied system. The degrees of the effects are different because of the differences between every applied system [2]. The non-linear modulation will occur which is caused by the defects such as spot and breach on the surface of optical component, which reduce the number of the components and service life [3]. So the system would not be effectively run by some of the serious defects on the components' surface [4]. Above all, we can see that the detection of defects on the components' surface is imperative in the quality test of precision component $[1,2]$ which has become more valuable.

The study of optical components based on surface flaw detection technology, including both domestic and foreign can be traced back to the 1980 s, generating a lot of methods for the flaw detection on the surface of lens [5]. It can be divided into two categories based on the main tool used to detect and the time spent; the traditional method of detection involved artificial optical instruments while the new

*Address correspondence to this author at the Nanpingzhonglu, Shenyang, China, Postcard: 110159; Tel: 15104000377;

E-mail: missjiangyueqiu@sina.com detection method involves modern computer vision. The distribution of the spectrum density is firstly determined by the reflection of infrared light on the optical components' surface as discussed in the literature [6]. According to this distribution, the optical element surface infrared spectroscopy is probed, and based on the characteristics and distribution of the infrared spectrum, the type and distribution of the component surface are determined. Literature [7] used the optical fiber transmission, a special signal, the frequency of the incident light and the reflected light signal contrast, for the realization of the surface cleanliness of the optical detecting element. Although traditional optical detection method is simple and can achieve a certain testing requirements, there are a lot of reference values, but there are significant deficiencies. The defects on the lens' surface can be detected by the imaging technique of coherent filtering shown in the literature [8]. Firstly, the low-frequency components in the imaging beams generated by the imaging system in a special diaphragm are filtered so that only the high-frequency component image containing information of the element surface flaws and the size according to the degree of shading of the image flaws and defects to achieve the integral intensity identifing disease, are obtained [9].

Overall, defects exist io the surface of the lens seriously affect the performance of precision optical components. Quickly and accurately detecting flaws in the surface of lens is the key to ensure production quality of lens. Defects such as scratches, dirt and pitting in the surface of lens were investigated in this paper. The key technology of machine vision inspection based on image recognition requires an indepth analysis and learning. Moreover, the programs of extraction and quantification based on image recognition in the surface of lens were studied. 


\section{ENHANCEMENT OF OPTICAL LENS' DEFECTS}

In this paper, the method introuduced in the article "A New Filtering Algorithm for Preserving Edges and Details of Digital Images" has been used [10-12].

\subsection{Modified Filtering Algorithm by the Edges and Details of the Image under Protected}

Each pixel of lens image can be attributed to three categories: (1) the target pixels; (2) the background pixels; and (3) the noise pixels that are produced by generating or transferring of the image. According to its place in the region, the noise pixels of the image are further divided into isolated point noise and non-isolated point noise. For those isolated point noise, its four neighboring pixels are used instead of the average gray value of the pixel itself; aand for non-isolated point noise, the noise of the pixel location on the image is used. Then the non-isolated point noise is broken down into two types of areas: edge area and non-edge area, correspoding to different positions in which the nonisolated noise pixels are processed by using a different template window. The major ideas are as follows:

As shown in Fig. (1), a template of non-directional and eight directional templates are chosen to determine whether the pixel to be processed is isolated. Cross in the center of each template is the symbol pixel to be processed and 1 is used to represent the neighboring pixels of the pixel to be processed. To calculate the absolute value of the difference between the pixel's gray value of the pending image and other pixels' gray value in each template without using the cross symbol itself, the pending pixels belonging to the isolated noise points or non-isolated noise points must be determined according to the absolute difference, and then different window templates must be used for filtering based on the judgment result. Following are the rules to deal with the pixel to be processed:

(1) To calculate the pixel's gray value of the pending image and the mean value of all the pixels in each template except the cross symbol of the pixel to be processed in Fig. (1), we can see the pixel to be processed as the isolated noise if the absolute value of the difference is very big. If the absolute value obtained is greater than each of a set threshold value, the pixel to be processed can be classified as an isolated point noise [13-15].

For the isolated noise in the image, its gray value can be used as the neighborhood of all other pixels, which can remove the isolated noise points from the image completely. Therefore, for isolated noise points in the image, the algorithm adopted four neighborhood templates to process as shown in Fig. (2).

(2) If the pixel is not the isolated noise point, then the difference of the gray value of the pixel to be processed and the mean value of all the gray pixels in templates except for the pixel of the cross symbol, is calculated. If the absolute difference value of the pixel to be processed and template $X$ shown in Fig. (1) is minimum, then the pixel to be processed pertains to the template $\mathrm{X}$ shown in Fig. (1). For non-isolated noise points, if $\mathrm{X}$ is the

\begin{tabular}{|l|l|l|l|l|}
\hline 0 & 0 & 0 & 0 & 0 \\
\hline 0 & 0 & 1 & 0 & 0 \\
\hline 0 & 1 & $\times$ & 1 & 0 \\
\hline 0 & 0 & 1 & 0 & 0 \\
\hline 0 & 0 & 0 & 0 & 0 \\
\hline
\end{tabular}

(a) No direction

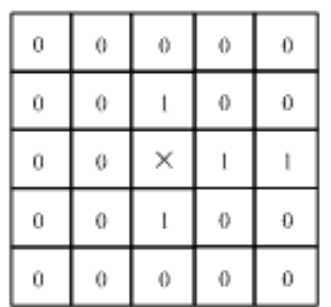

(d) Right

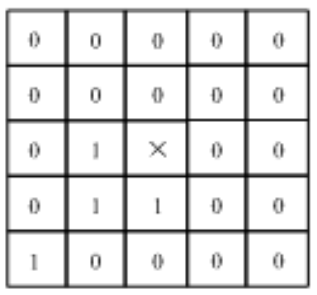

(g) Left bottom

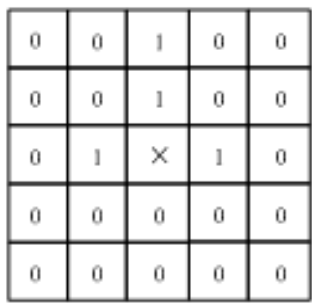

(b) Top

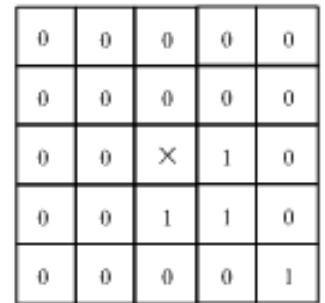

(e) Bottom right

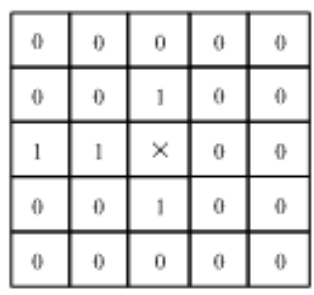

(h) Left

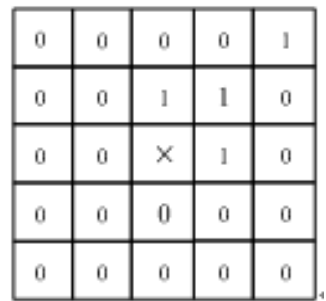

(c) Top right

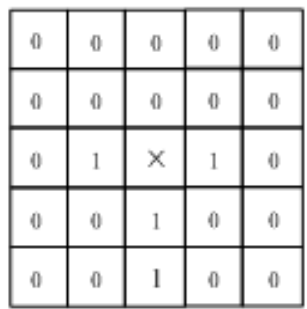

(f) Bottom

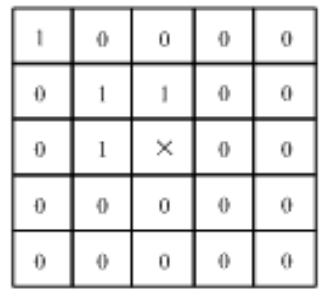

(i) Left top

Fig. (1). Templates are used to determine whether the pixel to be processed is pixel isolated. 
template as shown in Fig. (1) $[13,15]$, the gray value of the pixel to be processed is the mean value of all the pixels' gray template b as shown in Fig. (2) except for the cross symbol of pixels. If $\mathrm{X}$ is not the template as shown in Fig. (2), then we can judge the pixel to be processed to be non-isolated noise points of edge region or non-edge region according to the templates shown in Fig. (2).

\begin{tabular}{|c|c|c|}
\hline 0 & 1 & 0 \\
\hline 1 & $\times$ & 1 \\
\hline 0 & 1 & 0 \\
\hline
\end{tabular}

(a) Neighbors-domain

\begin{tabular}{|c|c|c|}
\hline 1 & 1 & 1 \\
\hline 1 & $\times$ & 1 \\
\hline 1 & 1 & 1 \\
\hline
\end{tabular}

(b) Eight neighborhood

Fig. (2). Templates are used to filter out the noise point.

(3) The gray value of the processing pixel and the mean gray value of all the pixels are compared in Fig. (3) in each template, except the cross symbol of the pixel to be processed.

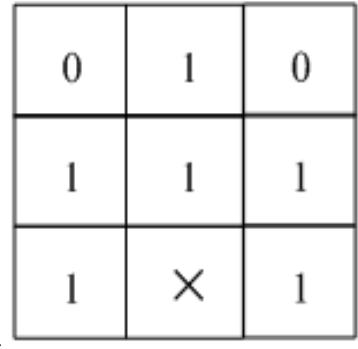

(a) Top

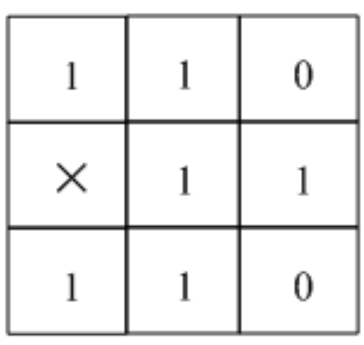

(c) Right

\begin{tabular}{|c|c|c|}
\hline 1 & $\times$ & 1 \\
\hline 1 & 1 & 1 \\
\hline 0 & 1 & 0 \\
\hline
\end{tabular}

(e ) Bottom

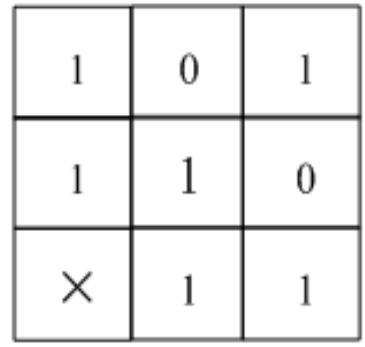

(b) Top right

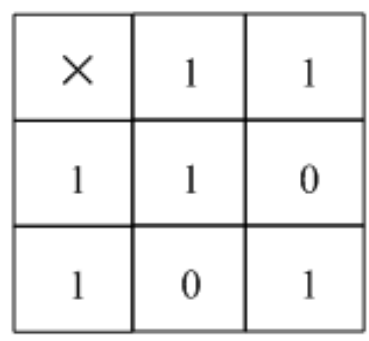

(d) Bottom right

\begin{tabular}{|l|l|l|}
\hline 1 & 1 & $\times$ \\
\hline 0 & 1 & 1 \\
\hline 1 & 0 & 1 \\
\hline
\end{tabular}

(f) Left bottom

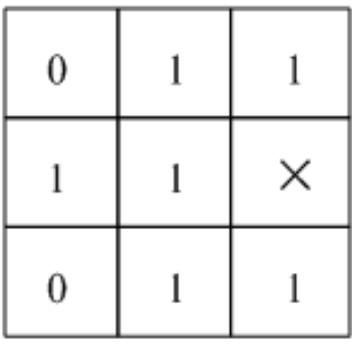

(g) Left

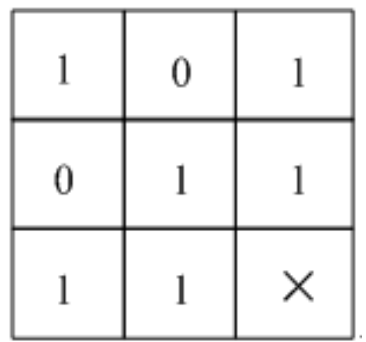

(h) Left top
Fig. (3). Templates are used to determine whether the pixel to be processed is non-isolated noise point of the edge region.

If the gray value of the pixel to be processed is far bigger or smaller than the mean gray value of all the pixels in Fig. (3) in each template, except the cross symbol of the p ixel to be processed (Fig. 1), then the pixel to be processed is seen as non-isolated point noise in edge region, otherwise it is non-isolated point noise in non-edge region. For non-isolated point noise in edge region, its gray value use the mean gray value of all the pixels in each template in Fig. (3) except for the cross symbol of the pixel to be processed. For nonisolated point noise in the non-edge region, its gray value use the mean gray value of all the pixels in template $\mathrm{x}$ except for the cross symbol of the pixel shown in Fig. (3).

\section{MODIFIED ALGORITHM OF IMAGE SEGMENTATION}

\subsection{Major Theory of Modified Two-dimensional Otsu Threshold Algorithms}

As Fig. (4) shows, two parallel lines $L_{1}$, and $L_{2}$ were drawn $^{\text {and }} L_{3}$ and $L_{4}$ on both sides of $L_{6}$.

Drawing a line perpendicular to $L_{6}$ passes by $(u, v)$, labelled as $L_{5}$. As the above figure shows, we use $L_{5}$ to distinguish between target and background. The formula $g=-f+u+v=-f+2 T, \quad(0 \leq u, v \leq L-1) \quad$ is used to express $L_{5}$. Between segment $g=f-u$ and $g=f+v$, when $g \geq-f+u+v$, we regard it as the background, when $g<-f+u+v$, we regard it as the target. And in that way, the histogram is divided into four new parts A、B、C、D; part $\mathrm{A}$ and part $\mathrm{C}$ are separately represented as the target and background, part $\mathrm{B}$ is regarded as the sidelines and part $\mathrm{D}$ is regarded as the noise area. To compare with the traditional two-dimensional Otsu threshold algorithm, only the pixels in part $\mathrm{A}$ and part $\mathrm{C}$ are involved in the calculation process, and the pixels in part B and part D are not involved in the calculation. However, the pixels nearby $L_{6}$ whose joint probabilities cannot be approximated to be zero, are involved in the calculation that will make up for the shortcoming of the traditional algorithms $[16,17]$. Averaging the grey value $f(x, y)$ and mean grey value of neighborhood $g(x, y)$, we segmented the image of lens by this average. $F(x, y)$ represents binary image: 


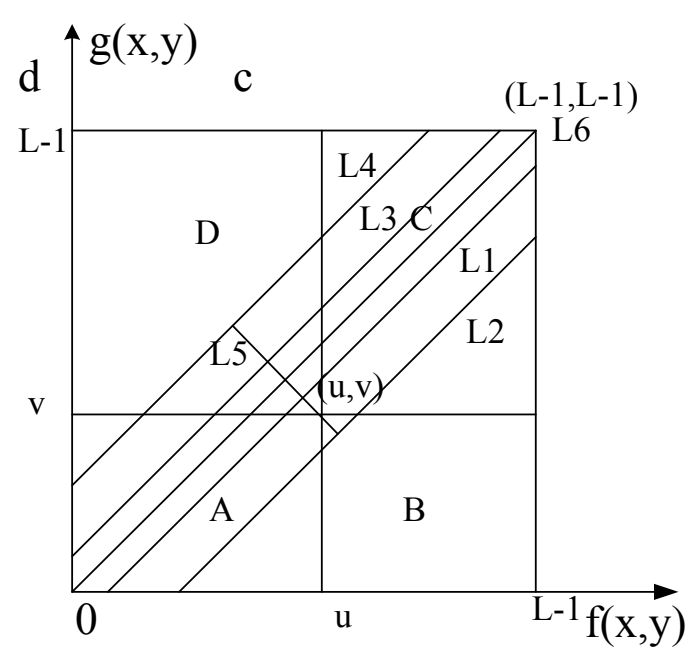

Fig. (4). Modified two-dimensional.

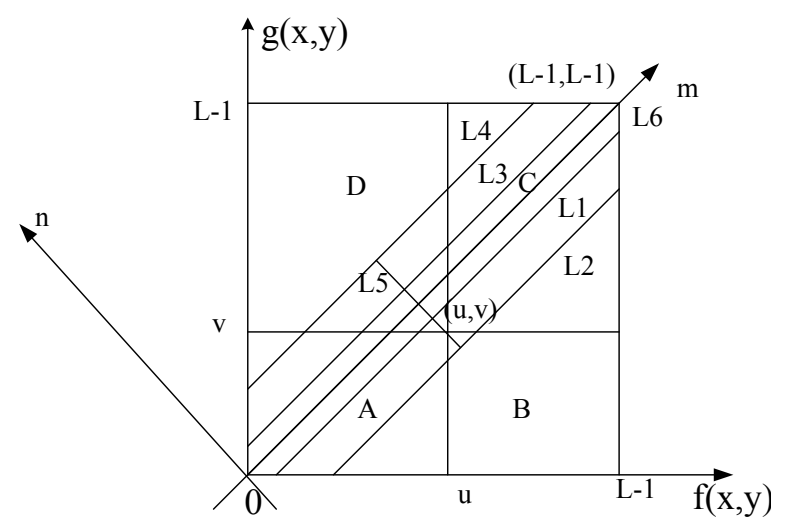

Fig. (5). Coordinate transformation modified.

$F(x, y)= \begin{cases}f(x, y), & \frac{1}{2}[f(x, y)+g(x, y)] \leq T ; \\ 255, & \frac{1}{2}[f(x, y)+g(x, y)>T .\end{cases}$

For two-dimensional Otsu threshold algorithms, we use the relative class instead of the variance and variance calculation method and the absolute class. The calculation reduces to be one-dimensional from two-dimensional threshold calculation; the specific method is as follows:

Fig. (5) shows contra rotation of the system $f-O-g$ around the origin of coordinates by $45^{\circ}$ to obtain a new set of coordinates $m-O-n$, where $(m, n)$ is the corresponding point in $m-O-n$ to $(x, y)$ in $f-O-g$. The conversion formula is as follows:

$\left\{\begin{array}{l}m=\frac{\sqrt{2}(x+y)}{2} \\ n=\frac{-\sqrt{2}(x-y)}{2}\end{array}\right.$

After conversion of the coordinate, the boundary of target and background represented as $g=-f+u+v=-f+2 T$ which can be converted into $m=T$. When $m \geq T$, it is regarded as target; when $m<T$ it is regarded as background. The target probability distribution $w_{0}$, and background probability distribution $w_{1}$ can be represented as:

$\left\{\begin{array}{c}w_{0}=\int_{T}^{\sqrt{2}(L-1)} \int_{-b / \sqrt{2}}^{a / \sqrt{2}} P(m, n) d m d n \\ w_{1}=\int_{0}^{T} \int_{-b / \sqrt{2}}^{a / \sqrt{2}} P(m, n) d m d n\end{array}\right.$

The target mean ${ }^{u_{0}}$ and the background mean $u_{1}$ can be represented as:

$\left\{\begin{array}{c}u_{0}=\left(u_{0 m}, u_{0 n}\right)=\left(\frac{1}{w_{0}} \int_{T}^{\sqrt{2}(L-1)} \int_{-b / \sqrt{2}}^{a / \sqrt{2}} m P(m, n) d m d n, \frac{1}{w_{1}} \int_{T}^{\sqrt{2}(L-1)} \int_{-b / \sqrt{2}}^{a / \sqrt{2}} n P(m, n) d m d n\right) \\ u_{1}=\left(u_{1 m}, u_{1 n}\right)=\left(\frac{1}{w_{0}} \int_{0}^{T} \int_{-b / \sqrt{2}}^{a / \sqrt{2}} m P(m, n) d m d n, \frac{1}{w_{1}} \int_{0}^{T} \int_{-b / \sqrt{2}}^{a / \sqrt{2}} n P(m, n) d m d n\right)\end{array}\right.$

The covariance matrix of target class $\delta_{0}$ and background class $\delta_{1}$ can be represented as:

$$
\left\{\begin{array}{c}
\delta_{0}=\frac{1}{w_{0}} \int_{T}^{\sqrt{2}(L-1)} \int_{-b / \sqrt{2}}^{a / \sqrt{2}}\left[(m, n)^{T}-u_{0}\right]\left[(m, n)^{T}-u_{0}\right]^{T} P(m, n) d m d n \\
\delta_{1}=\frac{1}{w_{1}} \int_{0}^{T} \int_{-b / \sqrt{2}}^{a / \sqrt{2}}\left[(m, n)^{T}-u_{1}\right]\left[(m, n)^{T}-u_{1}\right]^{T} P(m, n) d m d n
\end{array}\right.
$$

The covariance matrix between the classes shown above can be expressed as:

$$
\begin{aligned}
& \delta_{w}=w_{0} \delta_{0}+w_{1} \delta_{1}=\int_{0}^{T} \int_{-b / \sqrt{2}}^{a / \sqrt{2}}\left[(m, n)^{T}-u_{0}\right]\left[(m, n)^{T}-u_{0}\right]^{T} P(m, n) d m d n \\
& +\int_{T}^{\sqrt{2}(L-1)} \int_{-b / \sqrt{2}}^{a / \sqrt{2}}\left[(m, n)^{T}-u_{1}\right]\left[(m, n)^{T}-u_{1}\right]^{T} P(m, n) d m d n
\end{aligned}
$$

To trace $\operatorname{tr} \delta_{w}$ of the matrix $\delta_{w}$ taking the minimum, , and the value of $T$ satisfied $\operatorname{tr} \delta_{w}$ is the optimal threshold of $\delta_{w}$. So we can use $\operatorname{tr} \delta_{w}$ the variance within absolute clusters to take the place of $\delta_{w}$ in order to regard it as the level of dispersion test. After further transformation, we can obtain:

$$
\begin{aligned}
& \delta_{w}(T)=\delta_{0}+\delta_{1}=\frac{1}{w_{0}} \int_{0}^{T} \int_{-b / \sqrt{2}}^{a / \sqrt{2}}\left[(m, n)^{T}-u_{0}\right]\left[(m, n)^{T}-u_{0}\right]^{T} P(m, n) d m d n \\
& +\frac{1}{w_{1}} \int_{T}^{\sqrt{2}(L-1)} \int_{-b / \sqrt{2}}^{a / \sqrt{2}}\left[(m, n)^{T}-u_{1}\right]\left[(m, n)^{T}-u_{1}\right]^{T} P(m, n) d m d n
\end{aligned}
$$

The corresponding $\operatorname{tr} \delta_{w}(T)$ can be transformed into variance within relative clusters, as follows:

$$
\begin{aligned}
& \operatorname{tr} \delta_{w}(T)=\operatorname{tr} \delta_{0}+\operatorname{tr} \delta_{1}=\frac{1}{w_{0}} \int_{0}^{T} \int_{-b \sqrt{2}}^{a / \sqrt{2}}\left[\left(m-u_{0 m}\right)^{2}+\left(n-u_{0 n}\right)^{2}\right] P(m, n) d m d n \\
& +\frac{1}{w_{1}} \int_{T}^{\sqrt{2}(L-1)} \int_{-b / \sqrt{2}}^{a / \sqrt{2}}\left[\left(m-u_{1 m}\right)^{2}+\left(n-u_{1 n}\right)^{2}\right] P(m, n) d m d n
\end{aligned}
$$

To be compared with variance within absolute clusters, the descriptions of the target class and the background class by variance within relative clusters are more accurate. When $\operatorname{tr} \delta_{w}(T)$ is minimum, $T *$ is the best threshold, and then the image can be represented as: 


$$
F^{*}(m, n)= \begin{cases}f(m, n), & f(m, n)+g(m, n) \leq T^{*} \text { and } \frac{-\mathrm{a}}{\sqrt{2}} \leq f(m, n)-\mathrm{g}(\mathrm{m}, \mathrm{n}) \leq \frac{\mathrm{b}}{\sqrt{2}} ; \\ 255, & f(m, n)+g(m, n)>T^{*} \text { and } \frac{-\mathrm{a}}{\sqrt{2}} \leq f(m, n)-\mathrm{g}(m, n) \leq \frac{b}{\sqrt{2}} .\end{cases}
$$

After the transformation of the coordinate, $F^{*}(m, n)$, the standard of classification is relevant with $T *$. In that way, we transform two-dimensional threshold into onedimensional threshold to reduce the calculation and improve the efficiency of the image segmentation.

\section{RESEARCH ON AREAS' CALCULATION ALGORITHM OF LENS' DEFECTS}

\subsection{The Fundamental of the Algorithm}

The whole digital images are scanned in steps shown above. In the process of scanning traverse, the pixels of the defect target that appears are marked, for there is not only one target in the image of the lens, and the new target will appear continually. So the dynamic merge and mark for the adjacent target will continue till the scanning traverse is over and the new target appears. Only in that time, we can calculate the number of different targets' pixels, and by the resolution ratio, we can get the acreage of defects' target area.

\subsection{The Concrete Step of the Algorithm}

The concrete steps of algorithm to calculate the acreage of defects' target area in this paper are as follows:

Step 1: Enhancing and segmenting the lens image by the algorithms we have introduced above.

Step 2: Scanning traverse of the image to find out the center and the radius of the lens, locate the specific location and remove the interference of the border of the lens.

Step 3: Opening operation of the image, in the profile of the defects of lens that haven't changed significantly, and removing the interference of some small profile target.

Step 4: Closing operation of the image to connect with the adjacent target that has been disconnected, and smooth the profile of the defects of lens that significantly changed.

Step 5: Creating tag matrixes label 1 [], label 2 [], to resave labels.

Step 6: Scanning traverse of the image of lens from top to bottom, or left to right, and marking " 0 " when the grey level of current pixel is 255 . When the grey level of current pixel is 0 , the mark of the current pixel is decided by whether the pixels around were marked or not.

Step 7: Handling the pixels in the first line individually; if the pixels on the left were not mark, the label1 [] increased by " 1 ", or make the mark the same as the pixels on the left. The pixels in the first row are handled individually, if the pixels upon were not marked, the label1 [] increased by "1", or make the mark the same as the pixels upon;

Step 8: In this step, the general pixels that were not in the first line or row are dealt along with the marked image.
- If the left and upper images have been marked, but the two are not the same as the label, then the top of the label value can be seen as the current value, thus remarking the left by changing this line at the same time, and not the incorrect label adjacent to the pixels;

- If the left and the upper images have been marked, and the reference numerals are the same, then the current pixel can be left in any of the two reference numerals;

- If only the left is labeled, the label on the left can be taken;

- If the left and top are white areas, the label is recreated;

- The number of defects is stored in the final statistical defects by variable count;

Step 9: Scanning is again done to add up the number of defects and calculate the acreage of each defect;

Step 10: To mark from " 1 ", the number of each pixel is rearranged;

Step 11: To make binarization processing on each image, if the value of the pixel is smaller than 2, we can regard them as glitches and noises;

Step 12: In this step, the final statistics are stored including the number of storage defects, the defects of each pixel calculated based on the statistical results, and defects' size according to the resolution of the picture.

\section{IDENTIFICATION AND CLASSIFICATION OF THE LENS'S DEFECTS}

\subsection{Select of Excitation Function and Error Estimation's Method}

After the confirmation of input layer, output layer, hidden layer and train data, the error function completely depends on the excitation function, so it has important implications in the selection of excitation function for the astringency of network $[2,18]$. The input signal of another neuron in this system can be received by each of the artificial neurons in the BP neural network; different input corresponds to different weight, and the activated state of neuron desponds on the weight sum of all these weights. By the confirmation of $\mathrm{n}, \mathrm{q}, \mathrm{m}$ and train data, the error function also completely depends on the excitation function, so we can say that it is very important for the astringency of network on excitation function. By the demand of excitation function of BP algorithm, we choose $\mathrm{S}$ function as the excitation function. Although, by using neighborhood averaging method, we can excellently eliminate some noise as well, but it will blur the image and to handle the detailed image becomes difficult. We improved the neighborhood averaging method in the name of weighted average method. This made a difference in the weight of other pixels in the mode to the center pixel, and reduced the effects of image smoothing by other pixels in the mode. To compare it with the neighborhood averaging method, it improved the blooming phenomenon. Comparing these two methods, the selection type method combines the different statistic features of the target and background to makes the image smooth by choosing the windows which have minimum variance and reduces the blooming phenomenon as well as filters the noise. For the defects of lens, especially the spots, 
it does not make them smooth, the segmentation and judgment in the next step are influenced easily, so we should handle it carefully.

When the number of trained data and test data is limited, we usually use a part of the dates for training, the rest for testing as well as for handling which is called as the method of set. The 10-fold cross-validation method is used to estimate the technical error of machine learning when training data. The classified proportion of the trained data and the classified proportion of the whole data is equally divided into 10 parts randomly, to set one of them by ordinal turn, and participate in a training program based on ninetenths of the data. We use the data that we set above to calculate the rate of deviation. After training for 10 times, the average value of error rate's estimation is calculated and we can finally get the estimation of composition error.

\section{EXPERIMENTAL RESULTS}

\subsection{Image Enhancement of Optical lens' Defects}

The same image is handled in this paper by using mean, weighted mean, mask of select type and the algorithm of image enhancement respectively. The results are as shown in Fig. (6):

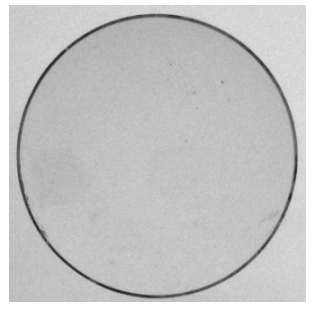

(a) select type mask

(c) neighborhood averaging

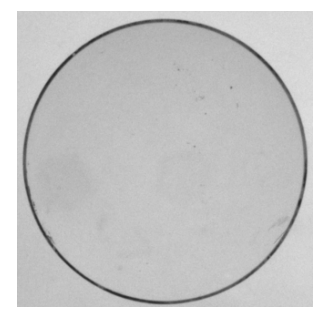

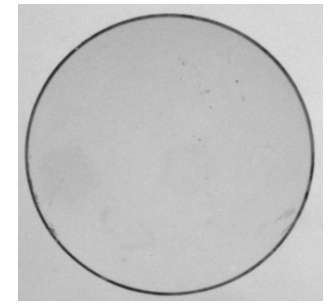

(b) weighted average

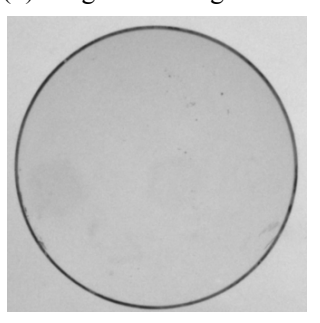

(d) this paper
Fig. (6). Comparison diagram of image smoothing algorithm's effect.

It is difficult to observe the difference between them subjectively, so we introduce the signal-to-noise ratio and peak signal to noise ratio. To make further on comparison, the formula of peak signal-to-noise ratio is as follows:
$P S N R=10 \log _{10}\left\{\frac{M \times N \times(G-1)^{2}}{\sum_{i=0}^{M-1} \sum_{j=0}^{N-1}[g(i, j)-f(i, j)]^{2}}\right\}$

By the dates shown in Table $\mathbf{1}$ we can see that the SNR and PSNR in the algorithm shown in this paper are much higher than others, and they have better smoothing effect. Based on the difference between the pixels,different measurements of the smoothing effect could be observed. As compared to the smoothing algorithm which did not separate the particular pixels, the algorithm we used in this paper can protect the outline of the image well and reduce the blooming phenomenon of the image.

\subsection{Image Segmentation of Lens's Defects}

Using the algorithm introduced in this paper, a large number of lens' images on the computer were handled, which runs IntelE7200/2.5 GHZ. We also obtained a good segmentation effect. Three lens' images were segmented by using one -dimensional Otsu threshold algorithm, traditional two-dimensional Otsu threshold algorithm and the improved two-dimensional Otsu threshold algorithm; the results are shown in Figs. (7-9):

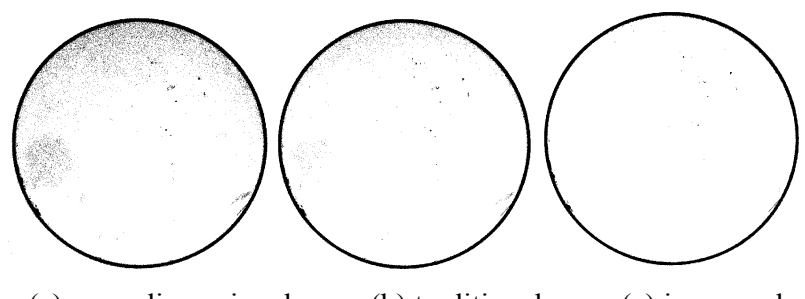

(a) one-dimensional

(b) traditional

(c) improved

Fig. (7). Comparison picture of image segmentation algorithms' effect of No.1 lens.

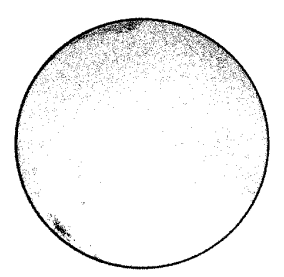

(a) one-dimensional

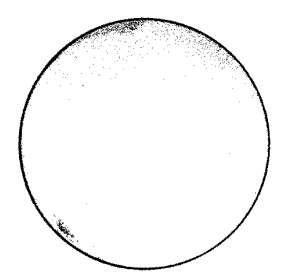

(b) traditional

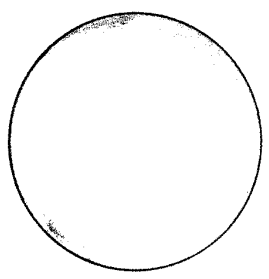

(c)improved
Fig. (8). Comparison picture of image segmentation algorithms' effect of No.2 lens.

Table 1. The test result of the smoothing algorithm above.

\begin{tabular}{|c|c|c|c|c|}
\hline The image & Mean value & weighted mean & Select type mask & This paper \\
\hline \hline SNR & 25.91 & 26.64 & 26.51 & 27.41 \\
\hline PSNR & 28.16 & 29.02 & 28.90 & 29.80 \\
\hline
\end{tabular}


Table 2. The comparison of the time used by one -dimensional, traditional two-dimensional and the improved.

\begin{tabular}{|c|c|c|c|c|}
\hline Test volume & Test image & This paper & Two-dimensional & One-dimensional \\
\hline \hline Time & No.1 & 23.864 & 128.373 & 17.944 \\
\hline $\mathrm{t}$ & No.2 & 30.142 & 124.455 & 20.745 \\
\hline Unit $: \mathrm{ms}$ & No.3 & 28.467 & 103.845 & 21.893 \\
\hline
\end{tabular}

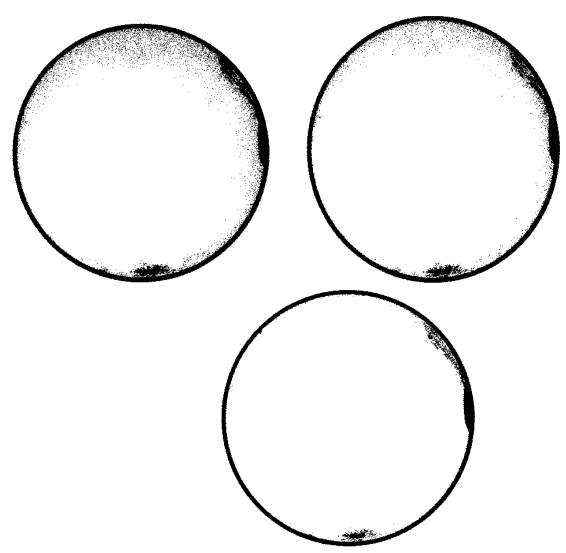

(a) one-dimensional (b)traditional (c)improved

Fig. (9). comparison picture of image segmentation algorithms' effect of No.3 lens.

In order to prove the performance of the algorithm, one dimensional Otsu threshold algorithm was compared with the traditional two-dimensional Otsu threshold algorithm and the improved two-dimensional Otsu threshold algorithm on the computer which runs IntelE7200/2.5GHZ; the results are as shown in Table 2.

By the comparison of time that the three algorithms required, it was observed that the time spent by the algorithm introduced in this paper is shorter than the time consumed by the traditional two-dimensional Otsu threshold algorithm and almost equal with the time consumed by onedimensional Otsu threshold algorithm. Grayscale of the image is represented as L. In the traditional two-dimensional Otsu threshold algorithm, we calculate each of the $u, v$ combination, to obtain the interclass variance. In each calculation, we need to accumulate $3 u v+3(L-u)(L-v)$ pixels; the time in total is shown as:

$D_{1}=\sum_{s=0}^{L-1} \sum_{t=0}^{L-1}[3 u v+3(L-u)(L-v)]=O\left(L^{4}\right)$

But,the algorithm studied in this paper only needed to multiply and accumulate the times of $\mathrm{L}$ after coordinating transformation, and coordinating transformation needs $9(L-1)(L-1)$ times multiplication and accumulation; so the times of multiplication and accumulation in total is shown as:

$D_{2}=\sum_{s=0}^{L-1} \sum_{t=0}^{L-1} L+9(L-1)(L-1)=O\left(L^{3}\right)$
As compared with the traditional two-dimensional Otsu threshold algorithm, the complexity of time has been reduced by an order of magnitude, making the speed of the processing faster than before. With respect to the complexity of space, when realizing this paper's algorithm, because of coordinating transformation by many times, there is a requirement of a larger space than that in the traditional two-dimensional Otsu threshold algorithm and onedimensional Otsu threshold algorithm; but by the improvement of computer technology, the demand of space can be satisfied by extending memory of computer. In order to get better processing effects, we can ignore these flaws.

\subsection{Areas' Calculation Algorithm of Lens's Defects}

Using the algorithm introduced in this paper; different lenses were analysed on the computer which runs IntelE7200/ 2.5GHZ. The results are shown in Fig. (10):

By the results, it can be observed that we can carry out calculation of the defects' outline faster and accurately only by one time scanning the image that has been preprocessed to achieve the desired treatment effect . The identification of defects' outline is very necessary for the extraction of the characteristic of a single defect's outline in the subsequent classification. If the number of defects in the lens is very large, it doesn't demand identifying the corresponding area and outline of the defects, we can obtain the result by using the algorithm introduced in this paper which will be much easier. Furthermore to make a little change in the algorithm , we can also express the encoding of the route of defects' outline to solve some problems in which the demands are much more difficult.

\subsection{Identification and Classification of the Lens' Defects}

Five images of lens defects were arbitrarily selected, where the number of defects in each image was different, and the rate of scratches and pitting was also different. Considering the absolute value of the difference between the numbers we obtained by visual inspection and BP neural network classification algorithm divided by the total number of test sample as misjudgment rate, we can obtain the result of classification and identification as shown in Table 3.

First we observed that the data of pitting in BP neural network was bigger than that in visual inspection. In the recognition of scratches, the results were equal to each other. Since the misjudgment rate of pitting is higher than that of scratches, that is because for some of the scratches, the shape of which is very coarse, it is difficult to judge whether these are scratches or pitting, which can lead to 


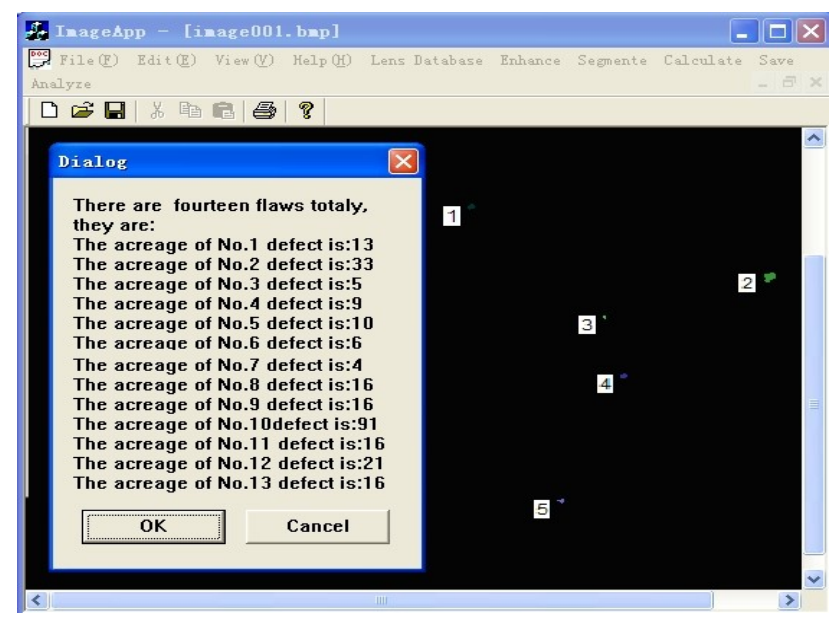

a. the calculate result of No.1 lens

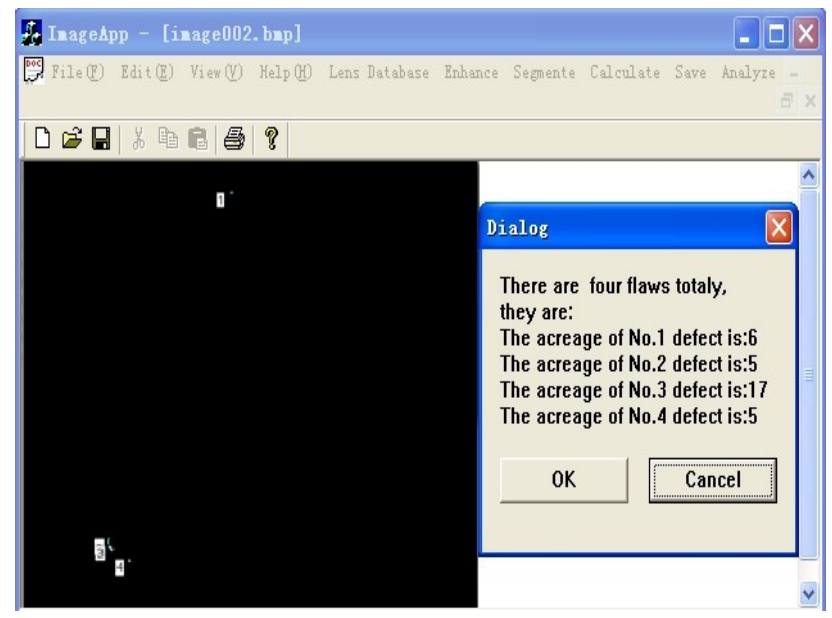

b. the calculate result of No.2 lens

Fig. (10). the calculate and demarcate results of lens's defects.

Table 3. Comparison result of optimization parameter in three kinds of method.

\begin{tabular}{|c|c|c|c|c|c|c|}
\hline \multirow{2}{*}{ pitting } & visual & 8 & 4 & 13 & 20 & 16 \\
\hline & Misjudgment & 0.10 & 0.25 & 0.18 & 0.21 & 0.4 \\
\hline scratches & Misjudgment & 0.10 & 0.25 & 0.06 & 0.04 & 0.2 \\
\hline
\end{tabular}

identification error. We can see in Table 3 within the margin of error that BP neural network can mainly recognize the scratches and pitting. On the other hand, BP neural network depends on machine recognition; therefore, the efficiency is much higher than visual inspection which is manual.

\section{CONCLUSION}

Based on an in-depth analysis of the key technologies of machine vision-based image recognition, combined with theoretical knowledge of digital image processing, pattern recognition and other related aspects, the authors conducted a research with the lens surface scratches, pitting and other defects as the object of the study. The paper put forward the scheme of division and quantization for processing of image recognition according to the lens surface defects. First, through the identification and classification of the noise pixels in different regions of the image, and choosing the corresponding window templates depending on their region, 
we removed the noise and protect the edges of the lens and any further damage to the image. Following this, combined with the characteristics of the lens flaws, we improved the two-dimensional Otsu threshold algorithm for decision threshold, and at the same time, the problem of lens flaws segmentation was properly solved. After that, the area of the communicated small target was calculated based on the improved mathematical morphology of pixel scanning, through which the area of the lens containing the flaw can also be calculated. Finally, the classification of scratches, and pitting was achieved through BP neural network algorithm. Experiments show certain achievements in the extraction of the lens surface scratches, pitting, dirt and other defects, by quantification and classification. For the study of lens scratches and pitting defects in the classification, however, the paper merely used BP neural network classification algorithm. In addition, this article only realized the classification and recognition of the scratches and pitting. The recognition of other defects such as the mouth, watermark, bubbles, etc has not been addressed. Therefore, further improvements are required for the study of classification and recognition of defects.

\section{CONFLICT OF INTEREST}

The author confirms that this article content has no conflict of interest.

\section{ACKNOWLEDGMENTS}

We would like to express our gratitude thanks to the Communication and Network Institute of Shenyang Ligong University for their invaluable suggestions to improve this paper. This work is supported by Liaoning Province Colleges and Universities Excellent Talents Support Program. (Grant No. LR201034), CALT Innovation Foundation (Grant No. 20130423), State Key Laboratory of Robotics Foundation, Shenyang Institute of Automation, Chinese Academy of Sciences (Grant No.2012017), and also supported by the Liaoning Province Educational Office Foundation of China (Grant No. L2011038).

\section{REFERENCES}

[1] B. Xiao, "Research on the key issues of automatic defects measurement on large fine optics", Master Dissertation of Zhejiang University 2010.
[2] L. Gao, "A Quick Method of Debris Detecting and Classifying for Oversize Optical Element", Master Dissertation of Southwest University of Science and Technology 2008.

[3] W. Xue, X. Zhijiang, S. Hongyan, and C. Ping, "Study on automatic flaw inspection system for large caliber precision optical surface", Chinese Journal of Scientific Instrument, vol. 2, pp. 4372, 2006.

[4] L. $\mathrm{Xu}$, and $\mathrm{Y}$. Yongying, "Error analysis of sub-aperture synthesis for detecting surface defects of optical componente", Journal of Optoelectronics Laser, vol. 8, pp. 223-232, 2008.

[5] X.K. Shi, M. Hua, "Automatic recognition and evaluation of micro - contaminant particles on ultra smooth optical substrates using image analysis method", Optics and Lasers in Engineering, vol. 41, no. 6, pp. 901-917, 2004.

[6] M. Druy, R.A. Bidduc, "Fiber optic non-contact reflectance probe for detection of contamination in pharmaceutical mixing vessels", SHE, vol. 3583, pp. 167-169, 1998.

[7] J.M. Lee, K.G. Watkins, "Chromatic modulation technique for inline surface monitoring and diagnostic", Journal of Cultural Heritage, vol. 1, pp. S311-S316, 2000.

[8] S. Weixing, "Surface defects of the optical elements measured by the coherent filter imaging system", Optical Technology, vol. 26, no.4, pp.361-365, 2000.

[9] W. Lei, M. Tao, K. Chengling, "Design of button cell automatic inspection system based on machine vision", Instrumentation Technology, vol. 28, no. 6, pp. 51-162, 2011.

[10] Y. Jiang, X. Fu, H. Gao, and S. Ma, "A new filtering algorithm for preserving edges and details of digital images", Sensors \& Transducers, pp. 1726-5479, 2014.

[11] Lu, P. Zhu, and Y. Cao, "The segmentation algorithm of improvement a two-dimensional Otsu and application research", 2010 2nd International Conference on Software Technology and Engineering, 2010.

[12] W.K. Liu, "Bridging scale method and its applications", Lecture Notes in Computational Science and Engineering, vol. 57, pp. 185205, 2007

[13] P. Tian, and L. Yinghua, "Color image enhancement based on improved intersecting cortical model", Journal of Computer Applications, vol. 32, no. 11, pp. 3153-3156, 2012.

[14] Z. Yujin, Image understanding and computer vision, Tsinghua University Press, pp. 60-163, 2000.

[15] Y. Jihong, "Edges and details keeping filtering algorithm for color images", Computer Engineering and Design, vol. 7, pp. 55-72, 2010.

[16] C. Bin, and T. Qichuan, "Improved fast adaptive Otsu segmentation algorithm \& its application", Application Research of Computers, vol. 29, no. 4, pp. 1572-1574, 2012.

[17] H. Zhibin, L. Tao, and Y. Kun, "Research on the sar image segmentation method based on improved two-dimension ostu arithmetic", Journal of Naval Aeronautical and Astronautically University, , vol. 25 , no. 2, pp. 153-157, 2010.

[18] P. Jishen, and D. Jing, "Method of control system sensor fault diagnosis based on RBF neural network", Modern Electronics Technique, vol. 12, pp. 179-1, 2009.

(C) Jiang et al.; Licensee Bentham Open.

This is an open access article licensed under the terms of the Creative Commons Attribution Non-Commercial License (http://creativecommons.org/licenses/by-nc/4.0/) which permits unrestricted, non-commercial use, distribution and reproduction in any medium, provided the work is properly cited. 